\title{
Internal Motions in a Fulleropyrrolidine Tertiary Amide with Axial Chirality
}

\author{
Giuseppe Borsato, ${ }^{\dagger}$ Federico Della Negra, ${ }^{\ddagger}$ Francesco Gasparrini, ${ }^{\S}$ Domenico Misiti, ${ }^{\S}$ Vittorio \\ Lucchini, ${ }^{*,}$ Giorgia Possamai, Claudio Villani, ${ }^{*, s}$ and Alfonso Zambon ${ }^{\dagger}$ \\ Dipartimento di Studi di Chimica e Tecnologia delle Sostanze Biologicamente Attive, Università di \\ Roma "La Sapienza", P.le A. Moro 5, 00185 Roma, Italy; Dipartimento di Scienze Ambientali, \\ Università di Venezia "Ca' Foscari”, Dorsoduro 2137, 30121 Venezia, Italy; Dipartimento di \\ Chimica Organica, Università di Padova, Via Marzolo 1, 35131 Padova, Italy \\ E-mail: claudio.villani@uniroma1.it; $\underline{\text { lucchini@unive.it }}$
}

\section{SUPPORTING INFORMATION}

Title page

Variable temperature NMR

Experimental and simulated dynamic ${ }^{1} \mathrm{H}$ NMR spectra of $\mathbf{1}$

Experimental and simulated dynamic ${ }^{1} \mathrm{H}$ NMR spectra of $\mathbf{2}$

Eyring plots for the enantiomerization and topomerization

processes in $\mathbf{1}$

Eyring plots for the enantiomerization and topomerization

processes in $\mathbf{2}$

Geometrically optimized structures and transition states for

$\mathrm{S} 7-\mathrm{S} 17$

interconversion (with imaginary frequencies) for s-trans-1, s-

cis-1, s-trans-2, s-cis-2, s-trans-3, s-cis-3, s-trans-4, s-cis-4. 
Variable temperature NMR

Temperature calibration was achieved by means of a calibrated thermocouple, which was inserted in the nitrogen stream just below the sample in the probe. Measurements with this apparatus were checked against those obtained by inserting a calibrated thermocouple into a solvent sample while it was in the probe. In addition, calibrations with the methanol standard (Amman, Meier, Merbach. J. Magn. Reson. 1982, 46, 319) were periodically performed. Temperature errors were no greater than $0.5 \mathrm{deg}$. Eyring plots for $\mathbf{1}$ and $\mathbf{2}$ are strictly linear and the high correlation coefficients obtained demonstrate a negligible effects of random errors. As a matter of facts, with such high correlation coefficients the introduction of the error for the dependent variable $(\operatorname{sigma}(\ln (\mathrm{k} / \mathrm{T}))$, calculated from the sigma(k) values and with sigma(T) set to 0.5$)$ does not alter significantly values and standard deviations for the slopes and intercepts. They remained unchanged also when we tested the system with an error artificially magnified 100 times.

Line shape simulations were performed with the DNMR5 program (D. S. Stephenson and G. Binsch, QCPE 11, 365 (1978)). Kinetic constants and the associated standard deviations were extracted using the same program. 


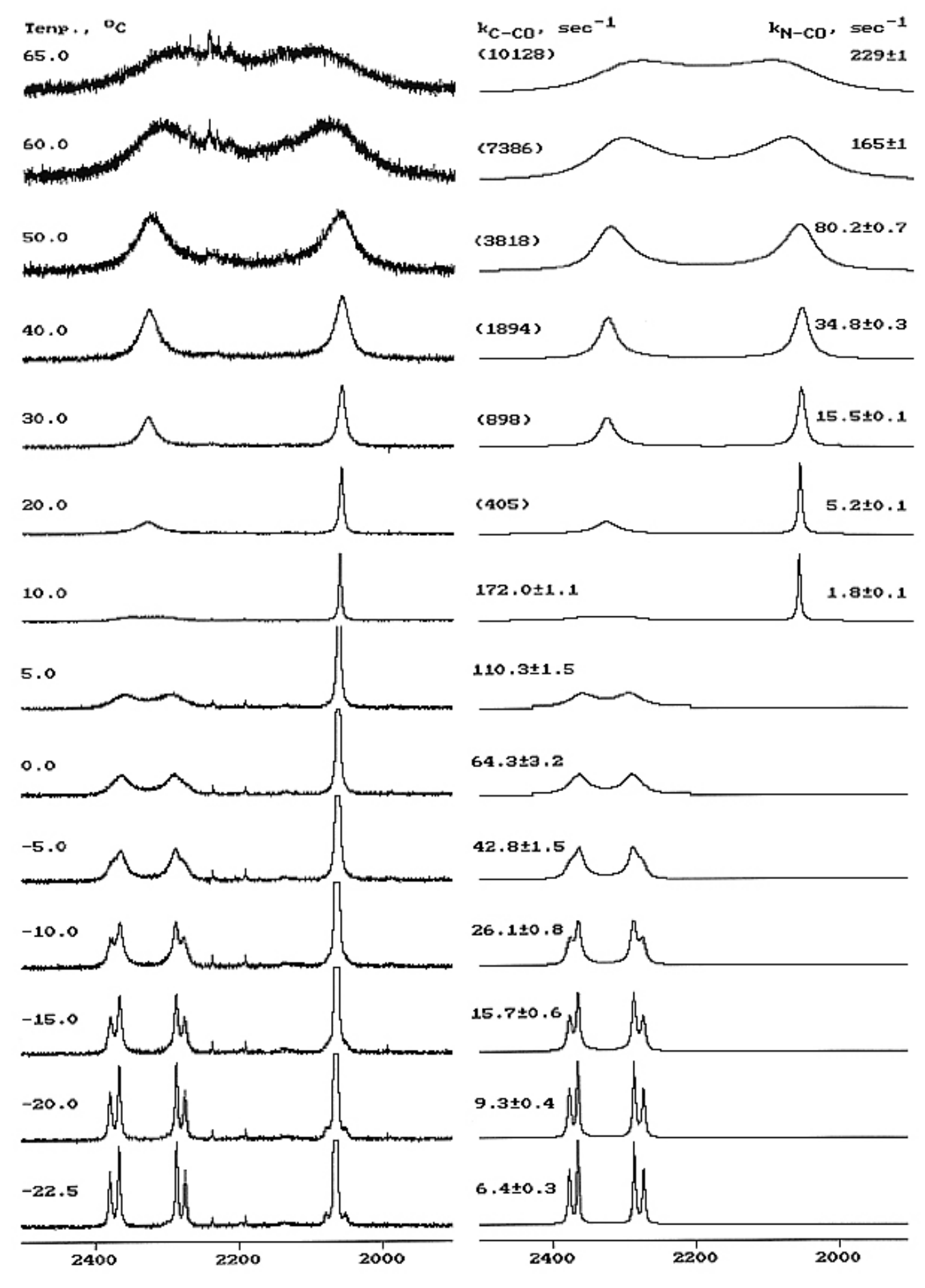

Experimental (left) and simulated (right) spectra at the reported selected temperatures, and associated kinetic costants (with standard errors) for the topomerization $\left(k_{\mathrm{N} \cdot \mathrm{CO}}\right)$ and enantiomerization $\left(k_{\mathrm{c} \cdot \mathrm{co}}\right)$ processes in $N$-1-naphthoyl fulleropyrrolidine 1. The simulations for enantiomerization were carried out on the low-field multiplet only. The values in parentheses are extrapolated from the low temperature data. 


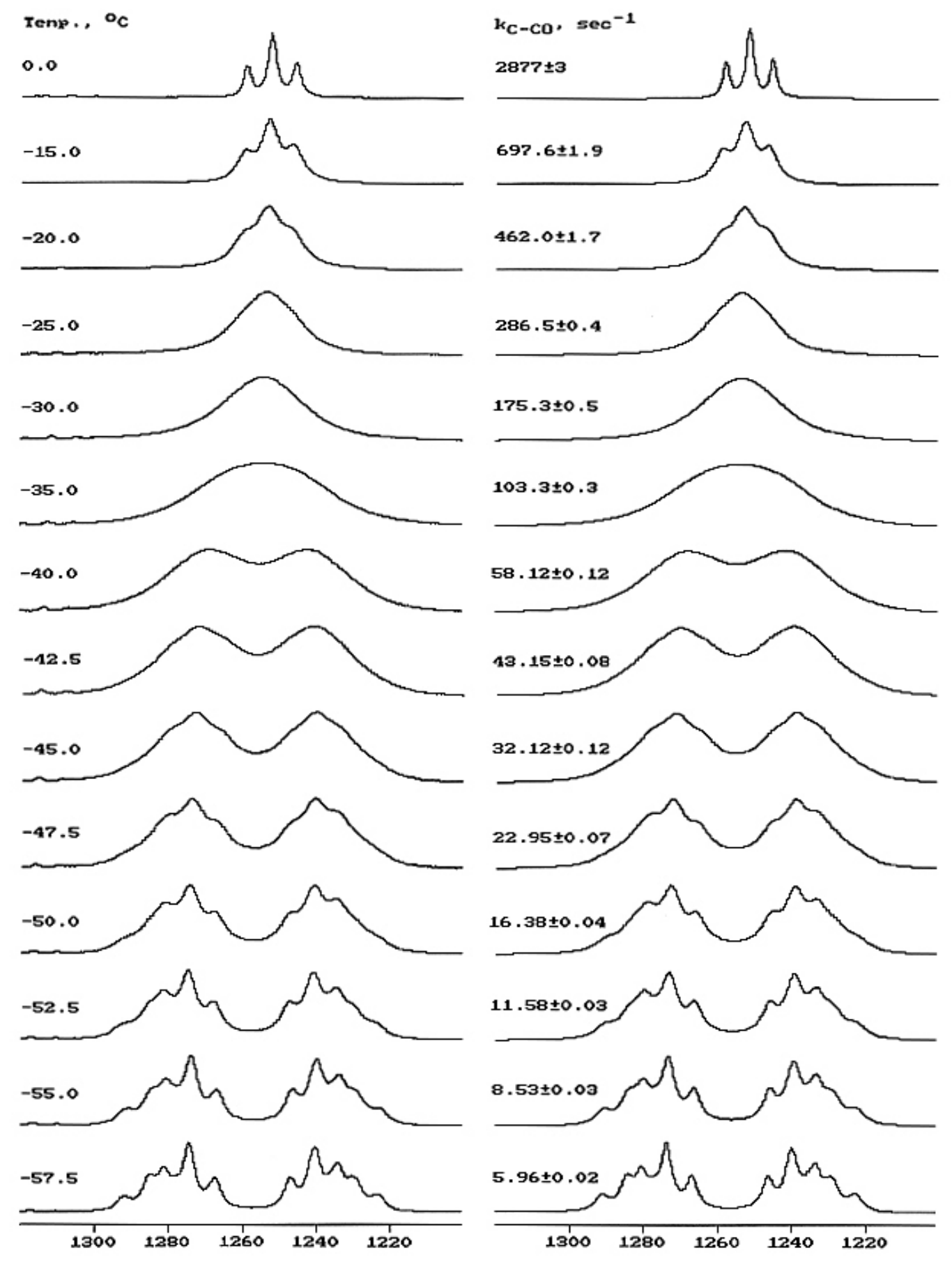

Experimental (left) and simulated (right) spectra at the reported selected temperatures, and associated kinetic costants (with standard errors) for the enantiomerization $\left(k_{\mathrm{C}-\mathrm{CO}}\right)$ processes in $N$ 1-naphthoyl pyrrolidine 2. The simulations were carried out on the multiplet resonating at $\delta 3.14$. 


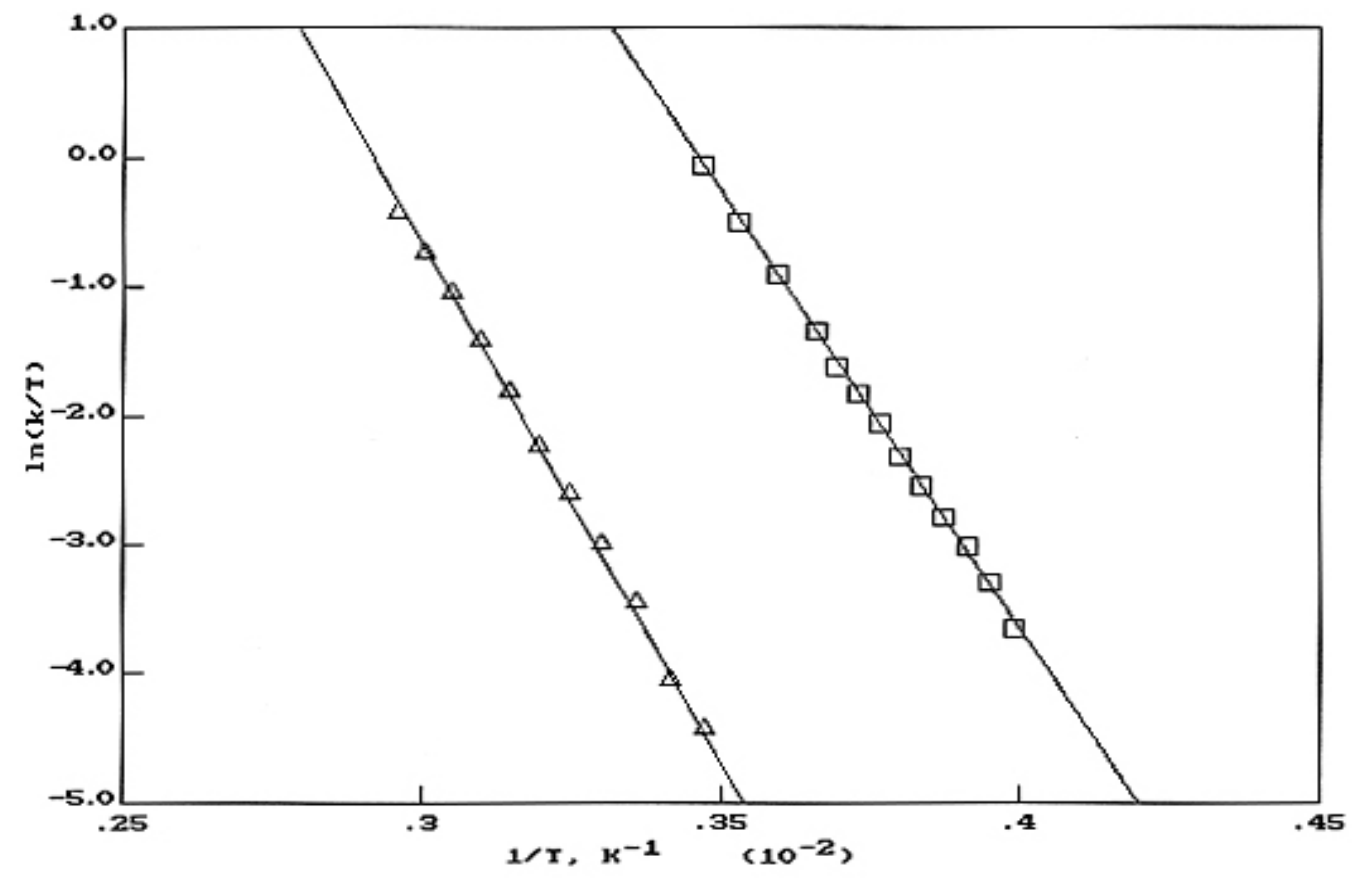

Eyring plots for the enantiomerization (i) and topomerization $(\Delta)$ processes in $N$-1-naphthoyl fulleropyrrolidine 1. Slope and intercepts are $-6804.9 \pm 48.2$ and $23.55 \pm 0.18$ (i) and $-8071.7 \pm$ 105.1 and $23.57 \pm 0.34(\Delta)$. Because of the high correlation coefficients $(0.999$ and 0.998$)$ the standard deviations are not significantly altered by the errors measured or calculated on temperatures and kinetic constants. 


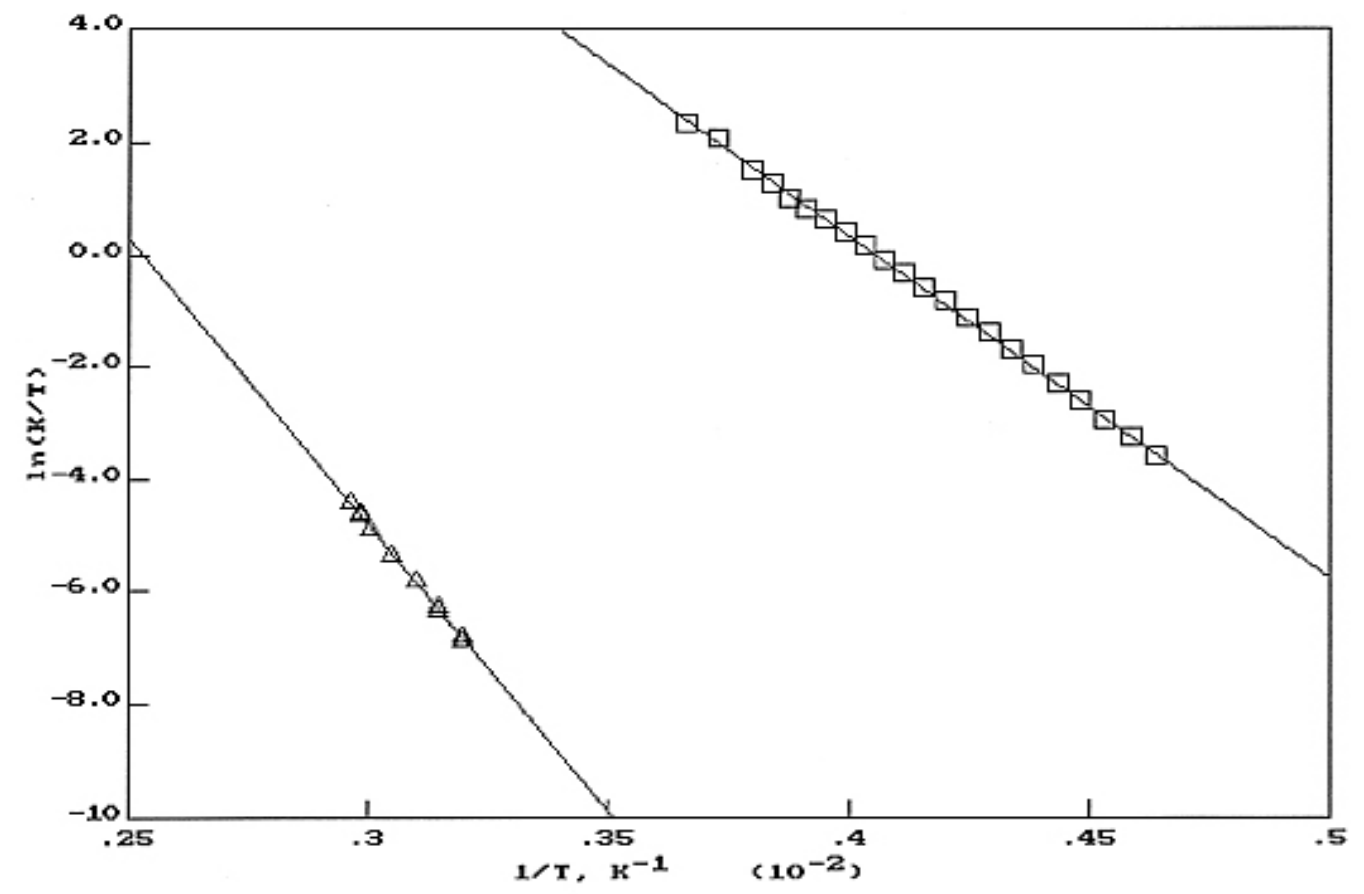

Eyring plots for the enantiomerization (i) and topomerization ( $\Delta$ ) processes in $N$-1-naphthoyl pyrrolidine 2. Slope and intercepts are $-6074.5 \pm 32.1$ and $24.62 \pm 0.13$ (i) and $-10202.3 \pm 79.5$ and $25.81 \pm 0.24(\Delta)$. Because of the high correlation coefficients $(0.999$ and 0.999$)$ the standard deviations are not significantly altered by by the errors measured or calculated on temperatures and kinetic constants. 
Structures 1: semi-empirical PM3 method for the fullerene spheroid (atoms signalled by L), ab initio $\mathrm{HF} / 631 \mathrm{G} * / / 631 \mathrm{G} *$ for the remnant (atoms signalled by H) .

Structures 2-4: ab initio $\mathrm{HF} / 631 \mathrm{G} * / / 631 \mathrm{G} *$.

Absolute energies (hartree):

s-trans-1: $\quad-629.17956272$

s-Cis-1: $\quad-629.17939437$

$\operatorname{TS}\left(\phi=180^{\circ}\right)-1: \quad-629.16288109$

Frequencies - $\quad-39.6225 \quad 21.3041 \quad 38.7284$

$\operatorname{TS}\left(\phi=90^{\circ}\right)-1: \quad-629.17910710$

Frequencies - $\quad-25.5881 \quad 9.0521 \quad 23.9503$

s-trans-2: $\quad-706.10069919$

s-Cis-2: $\quad-706.09887240$

$\operatorname{TS}\left(\phi=180^{\circ}\right)-2: \quad-706.08604814$

$60.1575 \quad 81.6242$

s-trans-3: $\quad-804.95183758$

S-Cis-3: $\quad-804.94997732$

$\operatorname{TS}\left(\phi=180^{\circ}\right)-3: \quad-804.93727399$

Frequencies - - $\quad-47.1439$

$61.5723 \quad 74.4904$

s-trans-4 : -704.91117700

s-cis-4: $\quad-704.91043485$

$\operatorname{TS}\left(\phi=180^{\circ}\right)-4: \quad-704.89582996$

Frequencies - - $\quad-62.2557 \quad 64.5862 \quad 95.2514$

Cartesian coordinates:

s-trans -1

01

C

C

C

C

C

C

C

C

C

C

C

C

C

C

C

C

C

C

C

C

C

C

C

C

C

C

C

$$
\begin{array}{r}
0.000000 \\
1.449229 \\
1.902904 \\
-0.454321 \\
0.724344 \\
-0.675782 \\
-1.852382 \\
-1.841928 \\
0.062177 \\
-0.667671 \\
2.133866 \\
1.430636 \\
2.307148 \\
3.312274 \\
3.334394 \\
3.025363 \\
3.750557 \\
4.211815 \\
3.032708 \\
3.767569 \\
-1.568448 \\
-1.565251 \\
-2.287807 \\
-2.288400 \\
-2.737875 \\
0.728529 \\
1.912061 \\
1.468495 \\
-0.446079
\end{array}
$$$$
0.000000
$$$$
0.000000 \mathrm{~L}
$$$$
0.000000
$$$$
0.000000 \mathrm{~L}
$$$$
\text { 1.385959 }
$$$$
0.000000 \mathrm{~L}
$$$$
\text { 1. } 385273
$$$$
0.009771 \mathrm{~L}
$$$$
\text { 2. } 242899
$$$$
0.010135 \mathrm{~L}
$$$$
\begin{array}{lll}
-0.959961 & 0.735937 \mathrm{~L}
\end{array}
$$$$
-0.584208 \quad 1.506934 \mathrm{~L}
$$$$
-1.358979 \quad 2.739752 \mathrm{~L}
$$$$
\begin{array}{lll}
-1.964339 & 1.482786 \mathrm{~L}
\end{array}
$$$$
-2.214493 \quad 2.735921 \mathrm{~L}
$$$$
-0.958660 \quad 0.731609 \mathrm{~L}
$$$$
-1.980681 \quad 1.472527 \mathrm{~L}
$$$$
-2.505636 \quad 2.611221 \mathrm{LH}
$$$$
-0.584041 \quad 1.491694 \mathrm{~L}
$$$$
-1.375467 \quad 2.700481 \mathrm{~L}
$$$$
\begin{array}{ll}
1.742799 & 0.723875 \mathrm{~L}
\end{array}
$$$$
0.731595 \quad 1.484350 \mathrm{~L}
$$$$
1.3389412 .716682 \mathrm{~L}
$$$$
2.976741 \quad 1.498995 \mathrm{~L}
$$$$
2.727419 \quad 2.732941 \mathrm{~L}
$$$$
1.740464 \quad 0.749558 \mathrm{~L}
$$$$
2.973183 \quad 1.525688 \mathrm{~L}
$$$$
2.726143 \quad 2.767561 \mathrm{~L}
$$$$
0.728838 \quad 1.514330 \mathrm{~L}
$$$$
1.340736 \quad 2.759279 \mathrm{~L}
$$$$
3.413417 \quad 0.749564 \mathrm{~L}
$$$$
3.789664 \quad 1.512891 \mathrm{~L}
$$

4.396577

$2.761179 \mathrm{~L}$

3. 787934

$1.526287 \mathrm{~L}$ 


\begin{tabular}{|c|c|c|c|c|c|}
\hline $\mathrm{C}$ & 0 & 0.010982 & 4.396709 & 2.769210 & $\mathrm{~L}$ \\
\hline $\mathrm{C}$ & 0 & 1.486055 & 1.947263 & 6.654460 & $\mathrm{~L}$ \\
\hline $\mathrm{C}$ & 0 & 1.931569 & 0.559216 & 6.636618 & $\mathrm{~L}$ \\
\hline $\mathrm{C}$ & 0 & 0.758289 & -0.291524 & 6.628012 & $\mathrm{~L}$ \\
\hline $\mathrm{C}$ & 0 & 0.028348 & 1.949517 & 6.662803 & $\mathrm{~L}$ \\
\hline C & 0 & 1.444189 & 3.912549 & 5.174743 & $\mathrm{~L}$ \\
\hline $\mathrm{C}$ & 0 & 2.166673 & 4.159562 & 3.932819 & $\mathrm{~L}$ \\
\hline $\mathrm{C}$ & 0 & 3.347056 & 2.520353 & 5.149301 & $\mathrm{~L}$ \\
\hline $\mathrm{C}$ & 0 & 3.344265 & 3.302215 & 3.918331 & $\mathrm{~L}$ \\
\hline $\mathrm{C}$ & 0 & 3.037899 & 0.192327 & 5.887344 & $\mathrm{~L}$ \\
\hline $\mathrm{C}$ & 0 & 3.764833 & 1.201510 & 5.130152 & $\mathrm{~L}$ \\
\hline $\mathrm{C}$ & 0 & 4.203410 & 0.591145 & 3.883109 & $\mathrm{~L}$ \\
\hline $\mathrm{C}$ & 0 & 3.025956 & -1.040301 & 5.118388 & $\mathrm{~L}$ \\
\hline $\mathrm{C}$ & 0 & 3.754381 & -0.790577 & 3.864337 & $\mathrm{~L}$ \\
\hline $\mathrm{C}$ & 0 & 0.762343 & -1.455828 & 5.874645 & $\mathrm{~L}$ \\
\hline $\mathrm{C}$ & 0 & 1.927033 & -1.856011 & 5.118558 & $\mathrm{~L}$ \\
\hline $\mathrm{C}$ & 0 & 1.518739 & -2.774018 & 3.965777 & $\mathrm{~L} \mathrm{H}$ \\
\hline $\mathrm{C}$ & 0 & -0.416102 & -1.830497 & 5.114565 & $\mathrm{~L}$ \\
\hline $\mathrm{C}$ & 0 & 0.023828 & -2.460989 & 3.890943 & $\mathrm{~L}$ \\
\hline $\mathrm{C}$ & 0 & -0.666537 & 2.908049 & 5.945051 & $\mathrm{~L}$ \\
\hline $\mathrm{C}$ & 0 & -1.850016 & 2.531813 & 5.181726 & $\mathrm{~L}$ \\
\hline $\mathrm{C}$ & 0 & -1.854684 & 3.306309 & 3.947456 & $\mathrm{~L}$ \\
\hline $\mathrm{C}$ & 0 & 0.059894 & 3.914961 & 5.182369 & $\mathrm{~L}$ \\
\hline $\mathrm{C}$ & 0 & -0.674837 & 4.162124 & 3.948062 & $\bar{L}$ \\
\hline $\mathrm{C}$ & 0 & -1.544970 & 0.207016 & 5.926396 & $\mathrm{~L}$ \\
\hline $\mathrm{C}$ & 0 & -1.543331 & -1.023241 & 5.143379 & $\mathrm{~L}$ \\
\hline $\mathrm{C}$ & 0 & -2.280654 & -0.779866 & 3.919633 & $\mathrm{~L}$ \\
\hline $\mathrm{C}$ & 0 & -2.279767 & 1.215738 & 5.173994 & $\mathrm{~L}$ \\
\hline $\mathrm{C}$ & 0 & -2.735731 & 0.605141 & 3.931315 & $\bar{L}$ \\
\hline $\mathrm{C}$ & 0 & 1.759515 & -4.236067 & 4.312668 & $\mathrm{H}$ \\
\hline $\mathrm{H}$ & 0 & 2.242138 & -4.339966 & 5.286863 & $\mathrm{H}$ \\
\hline $\mathrm{H}$ & 0 & 0.820925 & -4.791492 & 4.370048 & $\mathrm{H}$ \\
\hline $\mathrm{C}$ & 0 & 2.973397 & -3.823274 & 2.227919 & $\mathrm{H}$ \\
\hline $\mathrm{H}$ & 0 & 2.617706 & -4.136563 & 1. 242552 & $\mathrm{H}$ \\
\hline $\mathrm{H}$ & 0 & 4.055824 & -3.679295 & 2.170918 & $\mathrm{H}$ \\
\hline $\mathrm{N}$ & 0 & 2.616514 & -4.792828 & 3.258317 & $\mathrm{H}$ \\
\hline $\mathrm{C}$ & 0 & 2.925491 & -6.118447 & 3.428784 & $\mathrm{H}$ \\
\hline 0 & 0 & 2.461030 & -6.648869 & 4.407161 & $\mathrm{H}$ \\
\hline $\mathrm{C}$ & 0 & 3.891712 & -10.391052 & 4.022073 & $\mathrm{H}$ \\
\hline $\mathrm{C}$ & 0 & 3.582919 & -9.075486 & 3.857087 & $\mathrm{H}$ \\
\hline $\mathrm{C}$ & 0 & 4.068138 & -8.328690 & 2.740845 & $\mathrm{H}$ \\
\hline $\mathrm{C}$ & 0 & 4.884962 & -9.035374 & 1.821787 & $\mathrm{H}$ \\
\hline $\mathrm{C}$ & 0 & 5.186791 & -10.407302 & 2.025130 & $\mathrm{H}$ \\
\hline $\mathrm{C}$ & 0 & 4.704881 & -11.078722 & 3.099315 & $\mathrm{H}$ \\
\hline $\mathrm{H}$ & 0 & 3.503688 & -10.913858 & 4.878290 & $\mathrm{H}$ \\
\hline $\mathrm{H}$ & 0 & 2.965779 & -8.595107 & 4.577692 & $\mathrm{H}$ \\
\hline $\mathrm{C}$ & 0 & 3.795061 & -6.922125 & 2.476151 & $\mathrm{H}$ \\
\hline $\mathrm{C}$ & 0 & 5.418211 & -8.386776 & 0.679306 & $\mathrm{H}$ \\
\hline $\mathrm{H}$ & 0 & 5.810357 & -10.904058 & 1.302588 & $\mathrm{H}$ \\
\hline $\mathrm{H}$ & 0 & 4.936418 & -12.118125 & 3.248929 & $\mathrm{H}$ \\
\hline $\mathrm{C}$ & 0 & 5.154777 & -7.082619 & 0.449648 & $\mathrm{H}$ \\
\hline $\mathrm{C}$ & 0 & 4.343968 & -6.365462 & 1.354239 & $\mathrm{H}$ \\
\hline $\mathrm{H}$ & 0 & 6.033989 & -8.952474 & 0.002453 & $\mathrm{H}$ \\
\hline $\mathrm{H}$ & 0 & 5.552015 & -6.577477 & -0.412039 & $\mathrm{H}$ \\
\hline $\mathrm{H}$ & 0 & 4.179563 & -5.345419 & 1.110547 & $\mathrm{H}$ \\
\hline \multicolumn{6}{|c|}{ s-cis-1: } \\
\hline \multicolumn{6}{|c|}{01} \\
\hline $\mathrm{C}$ & 0 & -2.821312 & -0.757863 & 2.179635 & $\mathrm{~L}$ \\
\hline $\mathrm{C}$ & 0 & -1.796889 & -1.770569 & 2.020752 & $\mathrm{~L}$ \\
\hline $\mathrm{C}$ & 0 & -2.269938 & -2.745368 & 1.044734 & $\mathrm{~L}$ \\
\hline $\mathrm{C}$ & 0 & -3.932402 & -1.093376 & 1.297339 & $\mathrm{~L}$ \\
\hline $\mathrm{C}$ & 0 & -3.590137 & -2.323717 & 0.594501 & $\mathrm{~L}$ \\
\hline $\mathrm{C}$ & 0 & -2.444053 & 0.564133 & 2.352913 & $\mathrm{~L}$ \\
\hline
\end{tabular}




\begin{tabular}{|c|c|c|c|c|c|}
\hline $\mathrm{C}$ & 0 & -3.172505 & 1.619854 & 1.664048 & $\mathrm{~L}$ \\
\hline $\mathrm{C}$ & 0 & -2.211028 & 2.640796 & 1.272760 & $\mathrm{~L}$ \\
\hline $\mathrm{C}$ & 0 & -1.037380 & 0.925983 & 2.393164 & $\mathrm{~L}$ \\
\hline $\mathrm{C}$ & 0 & -0.891537 & 2.225708 & 1.717552 & $\mathrm{~L}$ \\
\hline $\mathrm{C}$ & 0 & -0.460350 & -1.402111 & 2.047982 & $\mathrm{~L}$ \\
\hline $\mathrm{C}$ & 0 & -0.065253 & -0.028922 & 2.263369 & $\mathrm{~L}$ \\
\hline $\mathrm{C}$ & 0 & 1.324724 & 0.216355 & 1.679591 & $\mathrm{~L} \mathrm{H}$ \\
\hline $\mathrm{C}$ & 0 & 0.473731 & -1.995252 & 1.108220 & $\mathrm{~L}$ \\
\hline $\mathrm{C}$ & 0 & 1.443206 & -0.986612 & 0.745118 & $\mathrm{~L}$ \\
\hline $\mathrm{C}$ & 0 & -1.380459 & -3.310493 & 0.149877 & $\mathrm{~L}$ \\
\hline $\mathrm{C}$ & 0 & 0.026761 & -2.929376 & 0.185988 & $\mathrm{~L}$ \\
\hline $\mathrm{C}$ & 0 & 0.515248 & -2.878795 & -1.177496 & $\mathrm{~L}$ \\
\hline $\mathrm{C}$ & 0 & -1.761157 & -3.486196 & -1.245706 & $\mathrm{~L}$ \\
\hline $\mathrm{C}$ & 0 & -0.587545 & -3.219511 & -2.068120 & $\mathrm{~L}$ \\
\hline $\mathrm{C}$ & 0 & -4.617792 & -0.088066 & 0.638340 & $\mathrm{~L}$ \\
\hline $\mathrm{C}$ & 0 & -5.000710 & -0.260258 & -0.756541 & $\mathrm{~L}$ \\
\hline $\mathrm{C}$ & 0 & -4.856350 & 1.026690 & -1.426074 & $\mathrm{~L}$ \\
\hline $\mathrm{C}$ & 0 & -4.230622 & 1.304677 & 0.830214 & $\mathrm{~L}$ \\
\hline $\mathrm{C}$ & 0 & -4.384228 & 1.994646 & -0.445190 & $\mathrm{~L}$ \\
\hline $\mathrm{C}$ & 0 & -3.951615 & -2.486577 & -0.732033 & $\mathrm{~L}$ \\
\hline $\mathrm{C}$ & 0 & -3.013865 & -3.082632 & -1.675533 & $\mathrm{~L}$ \\
\hline $\mathrm{C}$ & 0 & -3.158567 & -2.392692 & -2.950811 & $\mathrm{~L}$ \\
\hline $\mathrm{C}$ & 0 & -4.675531 & -1.428380 & -1.424454 & $\mathrm{~L}$ \\
\hline $\mathrm{C}$ & 0 & -4.186449 & -1.370951 & -2.796281 & $\mathrm{~L}$ \\
\hline $\mathrm{C}$ & 0 & -0.131617 & 0.844321 & -4.182376 & $\mathrm{~L}$ \\
\hline $\mathrm{C}$ & 0 & 0.971098 & 1.183493 & -3.291069 & $\mathrm{~L}$ \\
\hline $\mathrm{C}$ & 0 & 0.625016 & 2.402765 & -2.588237 & $\mathrm{~L}$ \\
\hline $\mathrm{C}$ & 0 & -1.160813 & 1.865343 & -4.029917 & $\mathrm{~L}$ \\
\hline $\mathrm{C}$ & 0 & -0.691291 & 2.832334 & -3.046037 & $\mathrm{~L}$ \\
\hline $\mathrm{C}$ & 0 & -0.491929 & -0.479611 & -4.361593 & $\mathrm{~L}$ \\
\hline $\mathrm{C}$ & 0 & -1.899419 & -0.852940 & -4.401266 & $\mathrm{~L}$ \\
\hline $\mathrm{C}$ & 0 & -2.043815 & -2.139870 & -3.731683 & $\mathrm{~L}$ \\
\hline $\mathrm{C}$ & 0 & 0.233639 & -1.533190 & -3.661734 & $\mathrm{~L}$ \\
\hline $\mathrm{C}$ & 0 & -0.725600 & -2.562240 & -3.277935 & $\mathrm{~L}$ \\
\hline $\mathrm{C}$ & 0 & 1.653243 & 0.184345 & -2.615500 & $\mathrm{~L}$ \\
\hline $\mathrm{C}$ & 0 & 1.276262 & -1.208158 & -2.812276 & $\mathrm{~L}$ \\
\hline $\mathrm{C}$ & 0 & 1.420056 & -1.892180 & -1.535060 & $\mathrm{~L}$ \\
\hline $\mathrm{C}$ & 0 & 2.032639 & 0.366704 & -1.224825 & $\mathrm{~L}$ \\
\hline $\mathrm{C}$ & 0 & 1.886481 & -0.932525 & -0.548696 & $\mathrm{~L}$ \\
\hline $\mathrm{C}$ & 0 & 0.983655 & 2.550701 & -1.256920 & $\mathrm{~L}$ \\
\hline $\mathrm{C}$ & 0 & 1.724323 & 1.523118 & -0.560785 & $\mathrm{~L}$ \\
\hline $\mathrm{C}$ & 0 & 1.483173 & 1.629849 & 0.943831 & $\mathrm{~L} \mathrm{H}$ \\
\hline $\mathrm{C}$ & 0 & 0.049797 & 3.144765 & -0.317502 & $\mathrm{~L}$ \\
\hline $\mathrm{C}$ & 0 & 0.216689 & 2.483038 & 0.956369 & $\mathrm{~L}$ \\
\hline $\mathrm{C}$ & 0 & -2.498585 & 1.510451 & -4.065190 & $\mathrm{~L}$ \\
\hline $\mathrm{C}$ & 0 & -3.436322 & 2.106548 & -3.121678 & $\mathrm{~L}$ \\
\hline $\mathrm{C}$ & 0 & -4.394038 & 1.080646 & -2.729798 & L \\
\hline $\mathrm{C}$ & 0 & -2.877068 & 0.116336 & -4.256181 & $\mathrm{~L}$ \\
\hline $\mathrm{C}$ & 0 & -4.049350 & -0.149321 & -3.431811 & $\mathrm{~L}$ \\
\hline $\mathrm{C}$ & 0 & -1.580698 & 3.397800 & -2.151301 & $\mathrm{~L}$ \\
\hline $\mathrm{C}$ & 0 & -1.198604 & 3.562178 & -0.753575 & $\mathrm{~L}$ \\
\hline $\mathrm{C}$ & 0 & -2.365573 & 3.304525 & 0.066262 & $\mathrm{~L}$ \\
\hline $\mathrm{C}$ & 0 & -2.989677 & 3.027948 & -2.189813 & $\mathrm{~L}$ \\
\hline $\mathrm{C}$ & 0 & -3.476403 & 2.970535 & -0.816949 & $\mathrm{~L}$ \\
\hline $\mathrm{C}$ & 0 & 2.638214 & 2.331879 & 1.650756 & $\mathrm{H}$ \\
\hline $\mathrm{H}$ & 0 & 3.343403 & 2.780718 & 0.950611 & $\mathrm{H}$ \\
\hline $\mathrm{H}$ & 0 & 2.267862 & 3.137179 & 2.295783 & $\mathrm{H}$ \\
\hline $\mathrm{C}$ & 0 & 2.395785 & 0.192799 & 2.766960 & $\mathrm{H}$ \\
\hline $\mathrm{H}$ & 0 & 1.933890 & 0.338670 & 3.751223 & $\mathrm{H}$ \\
\hline $\mathrm{H}$ & 0 & 2.921278 & -0.761656 & 2.786862 & $\mathrm{H}$ \\
\hline $\mathrm{N}$ & 0 & 3.297214 & 1.292210 & 2.433413 & $\mathrm{H}$ \\
\hline $\mathrm{C}$ & 0 & 4.446040 & 1.601181 & 3.089928 & $\mathrm{H}$ \\
\hline 0 & 0 & 4.950644 & 2.685974 & 2.991041 & $\mathrm{H}$ \\
\hline $\mathrm{C}$ & 0 & 6.234958 & -2.096399 & 1.585679 & $\mathrm{H}$ \\
\hline
\end{tabular}




$\begin{array}{lllrll}\mathrm{C} & 0 & 5.667708 & -0.949761 & 2.043511 & \mathrm{H} \\ \mathrm{C} & 0 & 5.626547 & -0.661157 & 3.436243 & \mathrm{H} \\ \mathrm{C} & 0 & 6.201333 & -1.587333 & 4.331941 & \mathrm{H} \\ \mathrm{C} & 0 & 6.782352 & -2.778514 & 3.821036 & \mathrm{H} \\ \mathrm{C} & 0 & 6.799028 & -3.029862 & 2.487158 & \mathrm{H} \\ \mathrm{H} & 0 & 6.261076 & -2.297083 & 0.529515 & \mathrm{H} \\ \mathrm{H} & 0 & 5.249530 & -0.247422 & 1.347238 \mathrm{H} \\ \mathrm{C} & 0 & 5.054359 & 0.536186 & 3.967154 & \mathrm{H} \\ \mathrm{C} & 0 & 6.198544 & -1.308559 & 5.724259 & \mathrm{H} \\ \mathrm{H} & 0 & 7.215413 & -3.479218 & 4.513242 & \mathrm{H} \\ \mathrm{H} & 0 & 7.243518 & -3.933043 & 2.109197 & \mathrm{H} \\ \mathrm{C} & 0 & 5.667478 & -0.154005 & 6.198835 & \mathrm{H} \\ \mathrm{C} & 0 & 5.100224 & 0.782881 & 5.304762 & \mathrm{H} \\ \mathrm{H} & 0 & 6.635231 & -2.025828 & 6.396896 \mathrm{H} \\ \mathrm{H} & 0 & 5.677727 & 0.060423 & 7.252176 \mathrm{H} \\ \mathrm{H} & 0 & 4.702412 & 1.705000 & 5.688009 & \mathrm{H}\end{array}$

$\operatorname{TS}\left(\phi=180^{\circ}\right)-1:$

\begin{tabular}{|c|c|c|}
\hline $\mathrm{C}$ & 0 & 1723817 \\
\hline $\mathrm{C}$ & 0 & 0.624905 \\
\hline $\mathrm{C}$ & 0 & 1.765984 \\
\hline $\mathrm{C}$ & 0 & 2.577549 \\
\hline $\mathrm{C}$ & 0 & 2.974551 \\
\hline $\mathrm{C}$ & 0 & 0.511953 \\
\hline $\mathrm{C}$ & 0 & 1.325308 \\
\hline $\mathrm{C}$ & 0 & 0.687023 \\
\hline C & 0 & -0.627368 \\
\hline $\mathrm{C}$ & 0 & -0.518266 \\
\hline $\mathrm{C}$ & 0 & -0.454386 \\
\hline $\mathrm{C}$ & 0 & -1.114518 \\
\hline $\mathrm{C}$ & 0 & -1.824030 \\
\hline C & 0 & -0.453619 \\
\hline $\mathrm{C}$ & 0 & -1.113273 \\
\hline C & 0 & 1.766713 \\
\hline C & 0 & 0.626398 \\
\hline $\mathrm{C}$ & 0 & 1.126549 \\
\hline $\mathrm{C}$ & 0 & 2.976057 \\
\hline $\mathrm{C}$ & 0 & 2.580294 \\
\hline C & 0 & 3.347860 \\
\hline $\mathrm{C}$ & 0 & 4.557562 \\
\hline $\mathrm{C}$ & 0 & 4.666241 \\
\hline C & 0 & 2.704965 \\
\hline $\mathrm{C}$ & 0 & 3.523829 \\
\hline C & 0 & 4.122999 \\
\hline C & 0 & 4.123763 \\
\hline $\mathrm{C}$ & 0 & 4.936171 \\
\hline C & 0 & 4.934932 \\
\hline $\mathrm{C}$ & 0 & 5.438643 \\
\hline C & 0 & 2.923367 \\
\hline $\mathrm{C}$ & 0 & 1.469472 \\
\hline $\mathrm{C}$ & 0 & 1.076455 \\
\hline C & 0 & 3.427914 \\
\hline $\mathrm{C}$ & 0 & 2.285284 \\
\hline C & 0 & 3.527022 \\
\hline $\mathrm{C}$ & 0 & 4.668665 \\
\hline $\mathrm{C}$ & 0 & 4.559982 \\
\hline C & 0 & 2.708626 \\
\hline $\mathrm{C}$ & 0 & 3.351047 \\
\hline C & 0 & 0.690199 \\
\hline C & 0 & 1.328960 \\
\hline $\mathrm{C}$ & 0 & 0.515131 \\
\hline $\mathrm{C}$ & 0 & -0.515880 \\
\hline $\mathrm{C}$ & 0 & -0.624961 \\
\hline
\end{tabular}

2.247311
2.931261
3.478149
2.357225
3.119100
1.073589
-0.045515
-1.272415
0.547147
-0.920402
2.403560
1.197757
0.519413
2.404864
1.199856
3.479497
2.933833
2.251955
3.121705
2.361890
1.289144
0.928244
-0.525562
0.057710
-1.063589
2.774865
2.776228
1.653688
1.651547
0.959047
-2.228812
-2.336702
-3.087389
-2.923299
-3.458412
-1.058185
-0.521439
0.932370
0.063906
1.294557
-1.267061
-0.039349
1.078951
0.916353

0

$2.595544 \mathrm{~L}$ $1.419291 \mathrm{~L}$ $0.694339 \mathrm{~L}$ $2.602611 \mathrm{~L}$ 1. $425024 \mathrm{~L}$ $3.005197 \mathrm{~L}$ $3.459151 \mathrm{~L}$ $3.003599 \mathrm{~L}$ $2.273111 \mathrm{~L}$ $2.272734 \mathrm{~L}$ $0.726708 \mathrm{~L}$ $1.172001 \mathrm{~L}$ $3.027518 \mathrm{~L}$ $-0.001420 \mathrm{~L} \mathrm{H}$ $-0.724743 \mathrm{~L}$ $-1.172875 \mathrm{~L}$ $-0.688137 \mathrm{~L}$ $-1.415251 \mathrm{~L}$ $-2.592180 \mathrm{~L}$ $-1.418171 \mathrm{~L}$ $-2.597517 \mathrm{~L}$ $2.300635 \mathrm{~L}$ $2.299444 \mathrm{~L}$ $3.471198 \mathrm{~L}$ $3.025668 \mathrm{~L}$ $0.732595 \mathrm{~L}$ $-0.725134 \mathrm{~L}$ $-1.175928 \mathrm{~L}$ $1.182248 \mathrm{~L}$ $0.002803 \mathrm{~L}$ $-2.601373 \mathrm{~L}$ $-2.596346 \mathrm{~L}$ $-1.420580 \mathrm{~L}$ $-1.423194 \mathrm{~L}$ $-0.694061 \mathrm{~L}$ $-3.025655 \mathrm{~L}$ $-2.297278 \mathrm{~L}$ $-2.295995 \mathrm{~L}$ $-3.470045 \mathrm{~L}$ $-3.023506 \mathrm{~L}$ $-3.006938 \mathrm{~L}$ $-3.459632 \mathrm{~L}$ $-3.004553 \mathrm{~L}$ $-2.276743 \mathrm{~L}$ $-2.274619 \mathrm{~L}$

64

S10 


\begin{tabular}{|c|c|c|c|c|c|}
\hline $\mathrm{C}$ & 0 & -0.070035 & -2.725951 & -0.729128 & $\mathrm{~L}$ \\
\hline $\mathrm{C}$ & 0 & -0.901062 & -1.630982 & -1.174947 & $\mathrm{~L}$ \\
\hline $\mathrm{C}$ & 0 & -1.704385 & -1.066185 & -0.002760 & L $\mathrm{H}$ \\
\hline $\mathrm{C}$ & 0 & -0.070836 & -2.727289 & 0.722363 & L \\
\hline $\mathrm{C}$ & 0 & -0.902250 & -1.633051 & 1.169242 & $\mathrm{~L}$ \\
\hline $\mathrm{C}$ & 0 & 4.511630 & -2.412220 & -0.729527 & L \\
\hline $\mathrm{C}$ & 0 & 4.510857 & -2.413557 & 0.728155 & $\mathrm{~L}$ \\
\hline $\mathrm{C}$ & 0 & 5.146788 & -1.182761 & 1.179843 & L \\
\hline $\mathrm{C}$ & 0 & 5.148035 & -1.180634 & -1.178349 & L \\
\hline $\mathrm{C}$ & 0 & 5.541707 & -0.420969 & 0.001636 & L \\
\hline $\mathrm{C}$ & 0 & 2.284495 & -3.459503 & 0.688443 & L \\
\hline $\mathrm{C}$ & 0 & 1.074928 & -3.089888 & 1.414379 & L \\
\hline $\mathrm{C}$ & 0 & 1.466721 & -2.341316 & 2.591905 & L \\
\hline $\mathrm{C}$ & 0 & 3.426386 & -2.925815 & 1.419757 & $\mathrm{~L}$ \\
\hline $\mathrm{C}$ & 0 & 2.920612 & -2.233443 & 2.598652 & L \\
\hline $\mathrm{C}$ & 0 & -3.130289 & -1.597872 & -0.003776 & $\mathrm{H}$ \\
\hline $\mathrm{H}$ & 0 & -3.322170 & -2.221463 & -0.879614 & $\mathrm{H}$ \\
\hline $\mathrm{H}$ & 0 & -3.322840 & -2.222868 & 0.870892 & $\mathrm{H}$ \\
\hline $\mathrm{C}$ & 0 & -3.314840 & 0.842624 & -0.002027 & $\mathrm{H}$ \\
\hline $\mathrm{H}$ & 0 & -3.557965 & 1.435427 & 0.884025 & $\mathrm{H}$ \\
\hline $\mathrm{H}$ & 0 & -3.556892 & 1.436390 & -0.887735 & $\mathrm{H}$ \\
\hline $\mathrm{N}$ & 0 & -4.023844 & -0.432692 & -0.003150 & $\mathrm{H}$ \\
\hline $\mathrm{C}$ & 0 & -5.358479 & -0.749774 & -0.002121 & $\mathrm{H}$ \\
\hline O & 0 & -5.623239 & -1.926292 & -0.001677 & $\mathrm{H}$ \\
\hline $\mathrm{C}$ & 0 & -9.646718 & -1.822952 & 0.002259 & $\mathrm{H}$ \\
\hline $\mathrm{C}$ & 0 & -8.321154 & -1.512848 & 0.000794 & $\mathrm{H}$ \\
\hline $\mathrm{C}$ & 0 & -7.871134 & -0.157628 & 0.000106 & $\mathrm{H}$ \\
\hline $\mathrm{C}$ & 0 & -8.881255 & 0.837825 & 0.000982 & $\mathrm{H}$ \\
\hline $\mathrm{C}$ & 0 & -10.254361 & 0.478362 & 0.002492 & $\mathrm{H}$ \\
\hline $\mathrm{C}$ & 0 & -10.638056 & -0.821520 & 0.003148 & $\mathrm{H}$ \\
\hline $\mathrm{H}$ & 0 & -9.939632 & -2.857939 & 0.002763 & $\mathrm{H}$ \\
\hline $\mathrm{H}$ & 0 & -7.606166 & -2.300050 & 0.000163 & $\mathrm{H}$ \\
\hline $\mathrm{C}$ & 0 & -6.480010 & 0.275765 & -0.001484 & $\mathrm{H}$ \\
\hline $\mathrm{C}$ & 0 & -8.541610 & 2.214392 & 0.000345 & $\mathrm{H}$ \\
\hline $\mathrm{H}$ & 0 & -10.987033 & 1.266308 & 0.003138 & $\mathrm{H}$ \\
\hline $\mathrm{H}$ & 0 & -11.679664 & -1.088735 & 0.004297 & $\mathrm{H}$ \\
\hline $\mathrm{C}$ & 0 & -7.247087 & 2.598026 & -0.001085 & $\mathrm{H}$ \\
\hline $\mathrm{C}$ & 0 & -6.230296 & 1.620199 & -0.001952 & $\mathrm{H}$ \\
\hline $\mathrm{H}$ & 0 & -9.332777 & 2.943351 & 0.001098 & $\mathrm{H}$ \\
\hline $\mathrm{H}$ & 0 & -6.974281 & 3.637760 & -0.001653 & $\mathrm{H}$ \\
\hline $\mathrm{H}$ & 0 & -5.236826 & 1.994255 & -0.003271 & $\mathrm{H}$ \\
\hline
\end{tabular}

$\operatorname{TS}\left(\phi=90^{\circ}\right)-1:$

$\begin{array}{lrr}0 \quad 1 & \\ C & 0 & 1.163190 \\ C & 0 & 0.313439 \\ C & 0 & 1.164408 \\ C & 0 & 2.546825 \\ C & 0 & 2.546943 \\ C & 0 & 0.845771 \\ C & 0 & 1.901555 \\ C & 0 & 1.371902 \\ C & 0 & -0.335694 \\ C & 0 & -0.006707 \\ C & 0 & -0.801477 \\ C & 0 & -1.152619 \\ C & 0 & -1.995535 \\ C & 0 & -1.128977 \\ C & 0 & -1.681445 \\ C & 0 & 0.852616 \\ C & 0 & -0.325791 \\ C & 0 & -0.006208 \\ C & 0 & 1.905915 \\ C & 0 & 1.374542\end{array}$

-0.905202
0.260394
1.444636
-0.452094
1.002858
-2.060815
-2.831657
-3.356623
-2.107147
-2.918378
0.199657
-1.015519
-0.689563
1.322088
0.797179
2.513700
2.452461
3.106232
3.201601
3.569214

$3.385013 \mathrm{~L}$ $3.245019 \mathrm{~L}$ $3.241667 \mathrm{~L}$ $3.460927 \mathrm{~L}$ $3.371507 \mathrm{~L}$ $2.689441 \mathrm{~L}$ $2.048481 \mathrm{~L}$ $0.797974 \mathrm{~L}$ $1.844880 \mathrm{~L}$ $0.661419 \mathrm{~L}$ $2.422778 \mathrm{~L}$ $1.724076 \mathrm{~L}$ $0.492791 \mathrm{~L} \mathrm{H} \quad 64$ $1.562361 \mathrm{~L}$ $0.334180 \mathrm{~L}$ $2.422398 \mathrm{~L}$ $1.565396 \mathrm{~L}$ $0.312100 \mathrm{~L}$ $1.686996 \mathrm{~L}$ $0.380339 \mathrm{~L}$
61 


\begin{tabular}{|c|c|c|c|c|c|}
\hline $\mathrm{C}$ & 0 & 3.546848 & -1.183098 & 2.844261 & $\mathrm{~L}$ \\
\hline $\mathrm{C}$ & 0 & 4.601881 & -0.499033 & 2.108780 & $\mathrm{~L}$ \\
\hline $\mathrm{C}$ & 0 & 4.928070 & -1.302001 & 0.936522 & $\mathrm{~L}$ \\
\hline $\mathrm{C}$ & 0 & 3.216121 & -2.406736 & 2.123300 & $\mathrm{~L}$ \\
\hline $\mathrm{C}$ & 0 & 4.074782 & -2.482579 & 0.947183 & $\mathrm{~L}$ \\
\hline $\mathrm{C}$ & 0 & 3.548083 & 1.653161 & 2.670297 & $\mathrm{~L}$ \\
\hline $\mathrm{C}$ & 0 & 3.219410 & 2.780521 & 1.806645 & $\mathrm{~L}$ \\
\hline $\mathrm{C}$ & 0 & 4.070504 & 2.706468 & 0.626202 & $\mathrm{~L}$ \\
\hline $\mathrm{C}$ & 0 & 4.602133 & 0.882653 & 2.023318 & $\mathrm{~L}$ \\
\hline $\mathrm{C}$ & 0 & 4.926139 & 1.534180 & 0.760408 & $\mathrm{~L}$ \\
\hline $\mathrm{C}$ & 0 & 2.404540 & 1.033678 & -3.321415 & $\mathrm{~L}$ \\
\hline $\mathrm{C}$ & 0 & 1.023396 & 0.571717 & -3.388331 & $\mathrm{~L}$ \\
\hline $\mathrm{C}$ & 0 & 1.025732 & -0.874335 & -3.291690 & $\mathrm{~L}$ \\
\hline $\mathrm{C}$ & 0 & 3.262332 & -0.137348 & -3.187708 & $\mathrm{~L}$ \\
\hline $\mathrm{C}$ & 0 & 2.409571 & -1.318932 & -3.173112 & $\mathrm{~L}$ \\
\hline $\mathrm{C}$ & 0 & 2.710700 & 2.197044 & -2.637616 & $\mathrm{~L}$ \\
\hline $\mathrm{C}$ & 0 & 3.891773 & 2.252995 & -1.786782 & $\mathrm{~L}$ \\
\hline $\mathrm{C}$ & 0 & 3.565588 & 3.055919 & -0.614480 & $\mathrm{~L}$ \\
\hline $\mathrm{C}$ & 0 & 1.651611 & 2.960607 & -1.988303 & $\mathrm{~L}$ \\
\hline $\mathrm{C}$ & 0 & 2.182797 & 3.496388 & -0.740435 & $\mathrm{~L}$ \\
\hline $\mathrm{C}$ & 0 & 0.017712 & 1.289413 & -2.761038 & $\mathrm{~L}$ \\
\hline $\mathrm{C}$ & 0 & 0.342441 & 2.517100 & -2.048831 & $\mathrm{~L}$ \\
\hline $\mathrm{C}$ & 0 & -0.508512 & 2.584943 & -0.869358 & $\mathrm{~L}$ \\
\hline $\mathrm{C}$ & 0 & -1.031306 & 0.597568 & -2.031603 & $\mathrm{~L}$ \\
\hline $\mathrm{C}$ & 0 & -1.360445 & 1.408012 & -0.847873 & $\mathrm{~L}$ \\
\hline $\mathrm{C}$ & 0 & 0.023453 & -1.514259 & -2.578392 & $\mathrm{~L}$ \\
\hline $\mathrm{C}$ & 0 & -1.044421 & -0.768713 & -1.951633 & $\mathrm{~L}$ \\
\hline $\mathrm{C}$ & 0 & -1.637055 & -1.571543 & -0.795055 & $\mathrm{~L}$ \\
\hline $\mathrm{C}$ & 0 & 0.350733 & -2.637342 & -1.718735 & $\mathrm{~L}$ \\
\hline $\mathrm{C}$ & 0 & -0.516433 & -2.582839 & -0.563877 & $\mathrm{~L}$ \\
\hline $\mathrm{C}$ & 0 & 4.383586 & -0.085189 & -2.377226 & $\mathrm{~L}$ \\
\hline $\mathrm{C}$ & 0 & 4.712223 & -1.212592 & -1.513591 & $\mathrm{~L}$ \\
\hline $\mathrm{C}$ & 0 & 5.238040 & -0.682784 & -0.262155 & $\mathrm{~L}$ \\
\hline $\mathrm{C}$ & 0 & 4.706421 & 1.141041 & -1.659293 & $\mathrm{~L}$ \\
\hline $\mathrm{C}$ & 0 & 5.235708 & 0.771969 & -0.352371 & $\mathrm{~L}$ \\
\hline $\mathrm{C}$ & 0 & 2.721329 & -2.388239 & -2.354122 & $\mathrm{~L}$ \\
\hline $\mathrm{C}$ & 0 & 1.664920 & -3.066885 & -1.612425 & $\mathrm{~L}$ \\
\hline $\mathrm{C}$ & 0 & 2.192743 & -3.439903 & -0.315271 & $\mathrm{~L}$ \\
\hline $\mathrm{C}$ & 0 & 3.903292 & -2.336144 & -1.503389 & $\mathrm{~L}$ \\
\hline $\mathrm{C}$ & 0 & 3.576762 & -2.987666 & -0.240913 & $\mathrm{~L}$ \\
\hline $\mathrm{C}$ & 0 & -2.938444 & -2.259249 & -1.198416 & $\mathrm{H}$ \\
\hline $\mathrm{H}$ & 0 & -3.138634 & -2.179014 & -2.267005 & $\mathrm{H}$ \\
\hline $\mathrm{H}$ & 0 & -2.903543 & -3.327301 & -0.954056 & $\mathrm{H}$ \\
\hline $\mathrm{C}$ & 0 & -3.480698 & -0.926162 & 0.759477 & $\mathrm{H}$ \\
\hline $\mathrm{H}$ & 0 & -3.605940 & -1.561056 & 1.644624 & $\mathrm{H}$ \\
\hline $\mathrm{H}$ & 0 & -4.006491 & 0.010118 & 0.948918 & $\mathrm{H}$ \\
\hline $\mathrm{N}$ & 0 & -3.982822 & -1.581772 & -0.441690 & $\mathrm{H}$ \\
\hline $\mathrm{C}$ & 0 & -5.278928 & -1.714753 & -0.810471 & $\mathrm{H}$ \\
\hline O & 0 & -5.602197 & -2.361067 & -1.769124 & $\mathrm{H}$ \\
\hline $\mathrm{C}$ & 0 & -6.443215 & 2.393615 & -1.476581 & $\mathrm{H}$ \\
\hline $\mathrm{C}$ & 0 & -6.073940 & 1.103794 & -1.262517 & $\mathrm{H}$ \\
\hline $\mathrm{C}$ & 0 & -6.665785 & 0.341884 & -0.217805 & $\mathrm{H}$ \\
\hline $\mathrm{C}$ & 0 & -7.650511 & 0.948041 & 0.589061 & $\mathrm{H}$ \\
\hline $\mathrm{C}$ & 0 & -8.013533 & 2.298123 & 0.339505 & $\mathrm{H}$ \\
\hline $\mathrm{C}$ & 0 & -7.427016 & 3.003050 & -0.661915 & $\mathrm{H}$ \\
\hline $\mathrm{H}$ & 0 & -5.990905 & 2.957185 & -2.272847 & $\mathrm{H}$ \\
\hline $\mathrm{H}$ & 0 & -5.337119 & 0.646944 & -1.897084 & $\mathrm{H}$ \\
\hline $\mathrm{C}$ & 0 & -6.302693 & -1.012723 & 0.047577 & $\mathrm{H}$ \\
\hline $\mathrm{C}$ & 0 & -8.259921 & 0.195875 & 1.628426 & $\mathrm{H}$ \\
\hline $\mathrm{H}$ & 0 & -8.765704 & 2.754121 & 0.959168 & $\mathrm{H}$ \\
\hline $\mathrm{H}$ & 0 & -7.709028 & 4.024577 & -0.844131 & $\mathrm{H}$ \\
\hline $\mathrm{C}$ & 0 & -7.905617 & -1.095039 & 1.848327 & $\mathrm{H}$ \\
\hline $\mathrm{C}$ & 0 & -6.913928 & -1.705837 & 1.044624 & $\mathrm{H}$ \\
\hline $\mathrm{H}$ & 0 & -9.012101 & 0.666712 & 2.236789 & $\mathrm{H}$ \\
\hline
\end{tabular}




$\begin{array}{llllll}\mathrm{H} & 0 & -8.373988 & -1.663930 & 2.631125 \mathrm{H} \\ \mathrm{H} & 0 & -6.648965 & -2.732559 & 1.225754 \mathrm{H}\end{array}$




$\begin{array}{rrrr}\mathrm{C} & 1.761811 & -1.684886 & 0.644570 \\ \mathrm{H} & 2.688372 & -2.705645 & -2.443240 \\ \mathrm{H} & 2.777332 & -3.432028 & -0.105412 \\ \mathrm{H} & 1.824148 & -2.001915 & 1.669445 \\ \mathrm{H} & -3.114565 & 1.490782 & 0.540696 \\ \mathrm{H} & -3.054126 & 0.057331 & 3.195392 \\ \mathrm{~F} & -4.128506 & -0.163642 & 0.945034 \\ \mathrm{H} & -3.412434 & 1.758307 & 2.954361\end{array}$

$$
\begin{aligned}
& \operatorname{TS}\left(\phi=180^{\circ}\right)-2: \\
& 01
\end{aligned}
$$

C
C
C
N
C
H
H
$\mathrm{H}$
$\mathrm{H}$
$\mathrm{H}$
$\mathrm{H}$
$\mathrm{H}$
$\mathrm{H}$
$\mathrm{C}$
$\mathrm{O}$
$\mathrm{C}$
$\mathrm{C}$
$\mathrm{C}$
$\mathrm{C}$
$\mathrm{C}$
$\mathrm{C}$
$\mathrm{H}$
$\mathrm{H}$
$\mathrm{C}$
$\mathrm{C}$
$\mathrm{H}$
$\mathrm{H}$
$\mathrm{C}$
$\mathrm{C}$
$\mathrm{H}$
$\mathrm{H}$
$\mathrm{H}$

$$
-3.267102
$$

C

C 


$\begin{array}{lrrr}\mathrm{H} & 1.729885 & 1.817591 & 0.370133 \\ \mathrm{C} & 0.607119 & -0.678926 & 0.184233 \\ \mathrm{C} & 1.749152 & -2.210982 & -1.853012 \\ \mathrm{H} & 3.617388 & -0.916653 & -3.276354 \\ \mathrm{H} & 4.391472 & 1.378641 & -2.926898 \\ \mathrm{C} & 0.783394 & -2.742456 & -1.063170 \\ \mathrm{C} & 0.200864 & -1.959093 & -0.040733 \\ \mathrm{H} & 2.192388 & -2.795116 & -2.640212 \\ \mathrm{H} & 0.451732 & -3.754523 & -1.208226 \\ \mathrm{H} & -0.565423 & -2.392832 & 0.576281 \\ \mathrm{H} & -3.237693 & 1.951497 & 0.212759 \\ \mathrm{H} & -3.928378 & 0.045839 & 2.454110 \\ \mathrm{~F} & -4.592610 & 0.512839 & 0.064922 \\ \mathrm{H} & -3.968335 & 1.800228 & 2.545721\end{array}$

s-cis-3:

\section{1}

C

C

C

$\mathrm{N}$

C

$\mathrm{H}$

$\mathrm{H}$

$\mathrm{H}$

$\mathrm{H}$

C

$\mathrm{O}$

C

C

C

C

C

C

$\mathrm{H}$

$\mathrm{H}$

C

C

$\mathrm{H}$

$\mathrm{H}$

C

C

$\mathrm{H}$

$\mathrm{H}$

$\mathrm{H}$

$\mathrm{H}$

$\mathrm{H}$

$\mathrm{H}$

$\operatorname{TS}\left(\phi=180^{\circ}\right)-3:$

$\mathrm{O}$
$\mathrm{C}$
$\mathrm{C}$
$\mathrm{N}$
$\mathrm{C}$
$\mathrm{H}$
$\mathrm{H}$
$\mathrm{H}$
$\mathrm{H}$
$\mathrm{C}$
$\mathrm{O}$
$\mathrm{C}$

$-3.199391$

$-2.812673$

$-1.429749$

$-0.892282$

$-1.897174$

$-1.486917$

$-0.785102$

$-1.856923$

$-1.796817$

0.322568

0.954284

1. 573455

1. 181992

1. 294386

1. 832202

2. 234049

2. 106425

1. 485478

0.806121

0.865213

1. 968512

2. 642194

2. 412639

1. 575293

1. 007255

2. 393137

1. 687281

0.697826

$-3.576369$

$-2.754150$

$-4.163414$

$-3.520141$
0.050928

0.678170

1. 259420

0.362105

$-0.559831$

2. 271502

1. 278257

$-0.639433$

$-1.555080$

0.636570

1.597238

1.888902

1. 504420

0.146255

$-0.788078$

$-0.351889$

0.948973

2. 919700

2. 231309

$-0.323171$

$-2.147362$

$-1.074031$

1. 271931

$-2.561638$

$-1.636530$

$-2.844917$

$-3.589352$

$-1.979601$

0.792359

$-0.109578$

$-0.908622$

1. 421054

1.651449

2. 979009

2. 688500

1. 662638

1. 147331

2. 304219

3. 555831

0.070651

1. 567440

1. 128871

1. 484728

$-2.831687$

$-1.588715$

$-1.179124$

$-2.089538$

$-3.379980$

$-3.744856$

$-3.124706$

$-0.896664$

0.102875

$-1.701791$

$-4.065133$

$-4.723647$

$-0.471840$

0.433620

$-2.402123$

$-0.177595$

1. 404493

0.958553

3. 723027

1. 788828

3. 325162

$$
\begin{array}{rr}
0.050928 & 1.651449 \\
0.678170 & 2.979009 \\
1.259420 & 2.688500 \\
0.362105 & 1.662638 \\
-0.559831 & 1.147331 \\
2.271502 & 2.304219 \\
1.278257 & 3.555831 \\
-0.639433 & 0.070651 \\
-1.555080 & 1.567440 \\
0.636570 & 1.128871 \\
1.597238 & 1.484728 \\
1.888902 & -2.831687
\end{array}
$$




\begin{tabular}{|c|c|c|c|}
\hline $\mathrm{C}$ & 1.181992 & 1.504420 & -1.588715 \\
\hline C & 1.294386 & 0.146255 & -1.179124 \\
\hline C & 1.832202 & -0.788078 & -2.089538 \\
\hline C & 2.234049 & -0.351889 & -3.379980 \\
\hline C & 2.106425 & 0.948973 & -3.744856 \\
\hline $\mathrm{H}$ & 1.485478 & 2.919700 & -3.124706 \\
\hline $\mathrm{H}$ & 0.806121 & 2.231309 & -0.896664 \\
\hline C & 0.865213 & -0.323171 & 0.102875 \\
\hline C & 1.968512 & -2.147362 & -1.701791 \\
\hline $\mathrm{H}$ & 2.642194 & -1.074031 & -4.065133 \\
\hline $\mathrm{H}$ & 2.412639 & 1.271931 & -4.723647 \\
\hline $\mathrm{C}$ & 1.575293 & -2.561638 & -0.471840 \\
\hline C & 1.007255 & -1.636530 & 0.433620 \\
\hline $\mathrm{H}$ & 2.393137 & -2.844917 & -2.402123 \\
\hline $\mathrm{H}$ & 1.687281 & -3.589352 & -0.177595 \\
\hline $\mathrm{H}$ & 0.697826 & -1.979601 & 1.404493 \\
\hline $\mathrm{H}$ & -3.576369 & 0.792359 & 0.958553 \\
\hline $\mathrm{H}$ & -2.754150 & -0.109578 & 3.723027 \\
\hline $\mathrm{F}$ & -4.163414 & -0.908622 & 1.788828 \\
\hline $\mathrm{H}$ & -3.520141 & 1.421054 & 3.325162 \\
\hline \multicolumn{4}{|c|}{ s-trans-4: } \\
\hline \multicolumn{4}{|c|}{$\begin{array}{ll}0 & 1\end{array}$} \\
\hline C & 0.665930 & -0.641135 & -3.419476 \\
\hline $\mathrm{N}$ & 0.765783 & -0.654270 & -1.961487 \\
\hline $\mathrm{C}$ & 2.112396 & -0.326392 & -1.491287 \\
\hline $\mathrm{H}$ & -0.056368 & 0.096646 & -3.751132 \\
\hline $\mathrm{H}$ & 0.335868 & -1.603897 & -3.796655 \\
\hline $\mathrm{H}$ & 2.114801 & 0.565202 & -0.871404 \\
\hline $\mathrm{H}$ & 2.545734 & -1.128888 & -0.904421 \\
\hline $\mathrm{C}$ & -0.367414 & -0.674993 & -1.224966 \\
\hline 0 & -1.454395 & -0.718353 & -1.740822 \\
\hline $\mathrm{C}$ & -1.981279 & 2.479191 & 1.262694 \\
\hline $\mathrm{C}$ & -1.479121 & 1.489538 & 0.478985 \\
\hline $\mathrm{C}$ & -0.794820 & 0.383026 & 1.055082 \\
\hline $\mathrm{C}$ & -0.661103 & 0.330081 & 2.458532 \\
\hline $\mathrm{C}$ & -1.199613 & 1.380290 & 3.248575 \\
\hline $\mathrm{C}$ & -1.838974 & 2.428107 & 2.669495 \\
\hline $\mathrm{H}$ & -2.499395 & 3.307105 & 0.812981 \\
\hline $\mathrm{H}$ & -1.617801 & 1.524834 & -0.583650 \\
\hline $\mathrm{C}$ & -0.217664 & -0.667724 & 0.275309 \\
\hline $\mathrm{C}$ & 0.002840 & -0.772897 & 3.057906 \\
\hline $\mathrm{H}$ & -1.091233 & 1.329919 & 4.317843 \\
\hline $\mathrm{H}$ & -2.244067 & 3.219373 & 3.274475 \\
\hline $\mathrm{C}$ & 0.519748 & -1.768788 & 2.296127 \\
\hline $\mathrm{C}$ & 0.414829 & -1.706041 & 0.887335 \\
\hline $\mathrm{H}$ & 0.085744 & -0.806962 & 4.130017 \\
\hline $\mathrm{H}$ & 1.013436 & -2.607476 & 2.752713 \\
\hline $\mathrm{H}$ & 0.831713 & -2.501487 & 0.295660 \\
\hline C & 2.852307 & -0.124513 & -2.785095 \\
\hline $\mathrm{H}$ & 3.895739 & 0.128269 & -2.808505 \\
\hline $\mathrm{C}$ & 2.071162 & -0.303520 & -3.828092 \\
\hline $\mathrm{H}$ & 2.365165 & -0.221296 & -4.857673 \\
\hline \multicolumn{4}{|c|}{ s-cis-4: } \\
\hline \multicolumn{4}{|c|}{$\begin{array}{ll}0 & 1\end{array}$} \\
\hline C & 0.079065 & -0.600171 & -3.395038 \\
\hline $\mathrm{N}$ & 0.132110 & -0.539401 & -1.935804 \\
\hline $\mathrm{C}$ & 1.401465 & -1.035990 & -1.402746 \\
\hline $\mathrm{H}$ & -0.132900 & 0.376664 & -3.817832 \\
\hline $\mathrm{H}$ & -0.707025 & -1.269439 & -3.727863 \\
\hline $\mathrm{H}$ & 1.904911 & -0.291984 & -0.796036 \\
\hline $\mathrm{H}$ & 1.258997 & -1.918065 & -0.784935 \\
\hline C & -1.016092 & -0.361469 & -1.241817 \\
\hline
\end{tabular}




\begin{tabular}{|c|c|c|c|}
\hline O & -2.073437 & -0.220120 & -1.797889 \\
\hline C & 1.088394 & 2.618503 & 1.186778 \\
\hline C & 0.443309 & 1.697692 & 0.424241 \\
\hline C & -0.227902 & 0.593759 & 1.021497 \\
\hline C & -0.228623 & 0.491570 & 2.428794 \\
\hline C & 0.462041 & 1.466944 & 3.197003 \\
\hline C & 1.105373 & 2.500299 & 2.596915 \\
\hline $\mathrm{H}$ & 1.585974 & 3.448814 & 0.718622 \\
\hline $\mathrm{H}$ & 0.429471 & 1.806164 & -0.644113 \\
\hline C & -0.935334 & -0.391006 & 0.264593 \\
\hline C & -0.932516 & -0.571542 & 3.052598 \\
\hline $\mathrm{H}$ & 0.459552 & 1.375199 & 4.26897 \\
\hline $\mathrm{H}$ & 1.621989 & 3.236023 & 3.186622 \\
\hline C & -1.624426 & -1.473171 & 2.311490 \\
\hline C & -1.635434 & -1.369432 & 0.901996 \\
\hline $\mathrm{H}$ & -0.919925 & -0.641659 & 4.126073 \\
\hline $\mathrm{H}$ & -2.170754 & -2.267482 & 2.786929 \\
\hline $\mathrm{H}$ & -2.202744 & -2.075206 & 0.323377 \\
\hline C & 2.166098 & -1.344565 & -2.660594 \\
\hline $\mathrm{H}$ & 3.173521 & -1.715595 & -2.635634 \\
\hline C & 1.452346 & -1.102225 & -3.738572 \\
\hline $\mathrm{H}$ & 1.775075 & -1.241843 & -4.753272 \\
\hline \multicolumn{4}{|c|}{$\operatorname{TS}\left(\phi=180^{\circ}\right)-4:$} \\
\hline $\mathrm{C}$ & -1.308801 & 0.000000 & 3.31444 \\
\hline $\mathrm{N}$ & -0.226383 & 0.000000 & 2.321458 \\
\hline $\mathrm{C}$ & 1.084153 & 0.000000 & 2.968643 \\
\hline $\mathrm{H}$ & -1.943133 & -0.870861 & 3.194745 \\
\hline $\mathrm{H}$ & -1.943133 & 0.870861 & 3.194745 \\
\hline $\mathrm{H}$ & 1.666565 & -0.879553 & 2.711064 \\
\hline $\mathrm{H}$ & 1.666564 & 0.879553 & 2.711064 \\
\hline $\mathrm{C}$ & -0.635537 & 0.000000 & 1.017919 \\
\hline 0 & -1.828462 & 0.000000 & 0.830395 \\
\hline $\mathrm{C}$ & -1.971385 & -0.000001 & -3.197900 \\
\hline $\mathrm{C}$ & -1.580455 & -0.000001 & -1.893939 \\
\hline C & -0.200184 & 0.000000 & -1.529023 \\
\hline C & 0.732082 & 0.000000 & -2.597140 \\
\hline C & 0.289004 & 0.000001 & -3.945454 \\
\hline C & -1.032118 & 0.000001 & -4.248271 \\
\hline $\mathrm{H}$ & -2.320410 & -0.000002 & -1.129598 \\
\hline C & 0.315550 & 0.000000 & -0.167306 \\
\hline C & 2.126714 & 0.000000 & -2.340113 \\
\hline $\mathrm{H}$ & 1.030134 & 0.000001 & -4.725381 \\
\hline $\mathrm{H}$ & -1.362614 & 0.000001 & -5.271493 \\
\hline $\mathrm{C}$ & 2.587194 & 0.000000 & -1.070879 \\
\hline C & 1.671721 & 0.000000 & 0.002947 \\
\hline $\mathrm{H}$ & 2.807004 & 0.000000 & -3.173396 \\
\hline $\mathrm{H}$ & 3.641425 & 0.000000 & -0.861355 \\
\hline $\mathrm{H}$ & 2.101086 & -0.000001 & 0.974605 \\
\hline $\mathrm{C}$ & 0.733491 & 0.000000 & 4.429942 \\
\hline $\mathrm{H}$ & 1.491575 & 0.000001 & 5.190626 \\
\hline C & -0.566384 & 0.000000 & 4.615245 \\
\hline $\mathrm{H}$ & -1.070793 & 0.000001 & 5.563276 \\
\hline $\mathrm{H}$ & -3.022146 & 0.000001 & -3.426944 \\
\hline
\end{tabular}

